\title{
Noch einmal: Das Komplementäre in der Medizin
}

Das muss jedem ungewöhnlich vorkommen: Im 6. Jahr ihres erfolgreichen Erscheinens führt eine wissenschaftliche Zeitschrift eine offenbar lange unterdrückte Diskussion über die richtige Interpretation ihres Namens!

Was ist Komplementärmedizin, was ihr Verhältnis und ihre Abgrenzung zur Naturheilkunde, und/oder in welcher Weise ergänzt sie diese? In den hierzu erschienenen drei Editorials vertraten die Disputanten etwas vereinfacht folgende Positionen: Bühring [1] führte in seinem Editorial aus, dass zwar «komplementär den Aussenseiter bezeichnet», dass aber «Komplementarität und Polarität hervorragende Modelle sind, das medizinische Denken sowie die Wahrnehmung und Beurteilung von Kranken und Gesunden, aber auch von Heilmitteln und -methoden der Natur lebendig zu erhalten». MeyerAbich [2] dagegen erklärt: «In dieser Situation nun scheint mir ein gütiges Geschick der Naturheilkunde den Gedanken der Komplementärmedizin beschert zu haben», was ihm letztlich in einer «komplementären Naturheilkunde» den Ausweg eröffnet. Schliesslich war es des Dritten (Linde [3]) Ziel, durch Interpretation des «Forschenden» in der Komplementärmedizin auf das Vorläufige, Unsichere, für Korrekturen Offene in der Debatte hinzuweisen und damit einen temporären Kompromiss zu ermöglichen.

Meyer-Abich [2] interessiert sich nicht dafür, wie der Begriff der Komplementarität in die Medizin gelangt ist, sondern er fordert uns auf, seinen naturphilosophischen Sinn in die Diskussion aufzunehmen. Allerdings meint auch er einen eng umschriebenen Sinn dieses Begriffs, nämlich die sogenannte Kopenhagener Deutung in der Physik. Dort hatten sich einige Phänomene der Quantenphysik seit Beginn der 20er Jahre als zunehmend rätselhaft dargestellt, bis sie dann durch Niels Bohrs zugegeben geniale Deutung eben als «komplementär» (insbesondere die Welle-Teilchen-Komplementarität) angesehen werden konnten. Es erscheint mir allerdings zweifelhaft, ob diese in der Physik bis heute gültige und nach Bohr noch erheblich erweiterte Sichtweise für die Medizin irgendeine Bedeutung hat.

Die Geschichte des Begriffs in der Medizin kann uns nicht ganz gleichgültig sein. Der medizinische Sprachgebrauch hierzulande ist bekanntlich in hohem Masse von dem im anglo-amerikanischen Raum abhängig. Von dort ist auch der Begriff der Komplementärmedizin gekommen. Deshalb hilft uns das deutschsprachige Verständnis des Begriffes (laut Brockhaus: Komplem'ent [lat] das, Ergänzung, Ergänzungsstück) nur bedingt weiter.

Im Grossbritannien der späten 70er Jahre bemühten sich einige prominente Mediziner auf Initiative von Prinz Charles, der sich von jeher in Architektur, Landwirtschaft und Heilkunde um naturnahe bzw. der Natur gerecht werdende Ansätze bemühte, um eine Sichtung derartiger Ansätze. Neben den traditionsreichen Schulen der britischen Homöopathie und der Osteopathie stiessen sie natürlich auch auf eine ganze Reihe anderer Therapie-Richtungen wie Phytotherapie, Akupunktur, Entspannungsverfahren usw., die damals in Grossbritannien keine ärztliche Anerkennung hatten. Das Komitee hatte sich unter anderem eine Namensgebung seines Arbeitsgebiets zur Aufgabe gesetzt und einigte sich nach langen Diskussionen auf «complementary medicine». Dass es dabei Bohrs Kopenhagener Deutung im Hinterkopf hatte, erscheint unwahrscheinlich, denn dem anglo-amerikanischen medizinischen Sprachverständnis sind die höheren Sphären der Physik ebenso fern wie dem deutschen. Anfangs verwechselten auch die Angelsachsen gelegentlich «complementary» mit «complimentary» und liefen Gefahr, an ein Kompliment (laut Brockhaus: [frz.] das, Artigkeit, Höflichkeitsbezeichnung, Schmeichelei; im Englischen auch ,compliment' gleich Gratifikation) zu denken.

Offenbar wollte man deutlich machen, dass es jenseits der allgemein anerkannten konventionellen Medizin (ein Begriff, der erstmalig wohl im britischen «Medical Act» von 1858 auftaucht) weitere Therapierichtungen gibt, die dem Anspruch Medizin zu sein gerecht werden. Das mag zwar trivial erscheinen, verdient jedoch deshalb festgehalten zu werden, da sich die deutschen Begriffe «Naturheilverfahren» und «Naturheilkunde» an diesem Punkt nicht positionieren lassen, was sie unter anderem für nichtärztliche Therapeuten völlig legitim verfügbar macht. Ähnlich schliesst übrigens das ebenfalls gebräuchliche «naturopathy» diese Therapeuten mit ein.

Diese Medizin hätte sich offenbar damals, zumindest im Vereinigten Königreich, nicht als eine neue Fachrichtung wie etwa die Gentherapie oder die Minimal Invasive Chirurgie etablieren lassen, die sich ihre Position durch innermedizinische Fortentwicklungen erkämpften. Mit der Wortschöpfung «complementary» konnte man zunächst jede Diffamierung vermeiden und blieb offen bezüglich der zu integrierenden Richtungen. Dies mag uns vielleicht etwas opportunistisch erscheinen, man muss jedoch bedenken, dass es eine Tradition ärztlich ausgeübter Naturheilverfahren im angloamerkanischen Raum nicht gibt - oder höchstens hinter vorgehaltener Hand. 1981 erschien die erste Monographie mit dem Begriff «complementary medicine» im Titel [4], seit Mitte der 80er Jahre haben ihm Übersichtsartikel in Lancet [5] und British Medical Journal [6] den Weg geebnet - was unter anderem dazu führte, dass

$\begin{array}{ll}\text { KARGER } & \text { ๑ 2000 S. Karger GmbH, Freiburg } \\ \text { Fax +497614520714 } & \text { Accessible online at: } \\ \begin{array}{l}\text { E-mail Information@Karger.de } \\ \text { www.karger.com }\end{array} & \text { www.karger.com/journals/fkm }\end{array}$

Dr. med. Rainer Stange

III. Innere Abteilung/Naturheilweisen

Turmstrasse 21

D-10559 Berlin (Deutschland) 
das bis dahin sehr gebräuchliche «alternative medicine» in Grossbritannien allmählich verschwand. In den USA hielt sich diese Sprachregelung noch länger, wurde mittlerweile aber zur Kompromissformel «complementary and alternative medicine» (Abkürzung CAM) geändert. Die weitere anglo-amerikanische Erfolgsgeschichte von «complementary medicine» mag nachträglich als eine Bestätigung dieser Strategie angesehen werden.

All dies hat zunächst wenig damit zu tun, auf welche Art von (Natur-)Wissenschaft sich unterschiedliche Therapierichtungen in der Medizin berufen. An diesem Punkt möchte ich Meyer-Abich heftig widersprechen, wenn er sagt, dass sich der «Schulmediziner» «auf die klassische, d.h. vor-quantentheroretische und insoweit überholte Naturwissenschaft» [2, S. 245] stütze.

Diskussionen über diese Form von medizinischem Selbstverständnis scheitern meines Erachtens regelmässig daran, dass, egal wo der Disputant selber steht, die Medizin, den grossen Naturwissenschaften vergleichbar, als ein wissenschaftlich homogenes Gebilde angesehen wird. Das ist sie aber mitnichten. Zwei Beispiele:

Ein Unfallchirurg, der mittels Osteosynthese eine Fraktur versorgt, wird sich auf die klassische Mechanik und die letztlich aus ihr abgeleiteten Prinzipien wie z.B. die Statik verlassen. Er weiss, dass Ingenieure, die Materialwahl und Formatierung seiner Hilfsmittel festgelegt haben, genauso dachten. Sein Natur- und Menschenbild ist wissenschaftstheroretisch das der vor-quantenmechanischen (nach Meyer-Abich «insoweit überholten») Naturwissenschaft. Er wird dabei allerdings sehr erfolgreich sein, während ein Abweichen von diesem Bild in Richtung auf irgendwelche «komplementäre» Deutungen von Frakturen ihn im Rahmen seiner Berufsausübung die Approbation kosten kann.

Wo steht dagegen ein Vertreter der Komplementärmedizin? Nehmen wir einen Akupunkteur! Immerhin kann er sich auf eine philosophische Tradition berufen, die vor etwa 2500 Jahren z. B. im Prinzip von Yin und Yang formal etwas Ähnliches wie die Komplementarität von Niels Bohr postuliert hat. Diese Philosophie sagt unter anderem aus, dass jedes Lebewesen und jedes seiner Phänomene wie etwa Symptome oder Krankheiten ständig Aspekte dieser beiden nur scheinbar gegensätzlichen, letztlich sich notwendig ergänzenden Qualitäten ausdrücken. Allerdings gibt es ganz entscheidende Unterschiede zwischen Bohrs Weltbild und dem der Traditionellen Chinesischen Medizin - das im übrigen über die Jahrhundert längst nicht so starr bleiben konnte, wie es heute im Westen gerne rezipiert wird. Ein guter chinesischer Arzt dürfte sich eher in der klassischen Position eines das Experiment nicht beeinflussenden und es «objektiv» wahrnehmenden Beobachters sehen. Und noch einen anderen Begriff der Komplementarität versucht Meyer-Abich in seinem Editorial einzuführen, möglicherweise ohne es zu wollen: «Die naturheilkundlichen Verfahren andererseits sind bei all ihrer Heterogenität gerade dort stark, wo die herrschende Medizin schwach ist.» [2, S. 245]. In diesem Ansatz gegenseitiger Ergänzung, völlig unabhängig von weltanschaulichen Unterschieden, könnte man etwas überspitzt den Naturheilverfahren die Rolle des Lückenbüssers zukommen lassen. Solche Positionen gibt es in der Tat, sie lassen sich kurzgefasst etwa so zusammenfassen: «tonangebende» Medizin für die akuten, Naturheilverfahren für die chronischen Krankheiten. So wird man aber weder dem Anspruch noch der gegenwärtigen Praxis gerecht, die doch von einer weitgehenden Durchdringung geprägt ist.

Letztlich geht es natürlich bei diesem meines Erachtens sehr lohnenden Streit um Begriffe wie «Komplementärmedizin» und «Naturheilkunde» um sehr viel mehr, nämlich um unsere Weltbilder. Über die zu streiten ist ebenso legitim wie ausser Mode gekommen. Die moderne Naturwissenschaft ist daran nicht ganz unschuldig, stellt sie sich selbst doch am liebsten in selbst auferlegter Abstinenz gegenüber dem Gebrauch von Weltbildern dar. Und da die meisten Aussenstehenden sie eh nicht mehr verstehen, gelingt diese Tarnung recht gut.

Zurück zu den Ärzten, den naturwissenschaftlichen «underdogs»: Ein Teil ihrer Probleme liegt darin begründet, dass sie nach aussen eine geschlossene Berufsgruppe darstellen, die durch vielerlei äusserliche Standards wie Gegenstandskatalog und Staatsexamen, Standesrecht, Zwangsmitgliedschaft in Kammern, Weiterbildungsordnungen usw. in eine homogene Gruppe gepresst werden. Wissenssoziologisch stellen Ärzte, gemessen an ihren «Denkstil» alles andere als ein «Denkkollektiv» etwa im Sinne Flecks [7] dar. Im Vergleich zu Naturwissenschaftlern findet sich selbst unter «naturwissenschaftlich orientierten» Ärzten eine Vielzahl von Modellbildungen. Meyer-Abichs Ansatz dagegen unterstellt paritätische Partner - die Ärzteschaft im Dialog mit seinen eigenen, wesentlich homogeneren naturwissenschaftlichen Denkkollektiven. Ob aber Ärzte sich überhaupt in vergleichbarem Masse vereinheitlichen lassen und ob dies überhaupt wünschenswert ist, möchte ich erheblich bezweifeln.

Medizinische wie nichtmedizinische Weltbilder der «komplementären» Ärzte sind bislang nicht untersucht worden. Ich riskiere einige Thesen: Diese Ärzte sind für ihre Patienten engagiert bis überengagiert, erinnern sich eher an das primum nil nocere, sind offen für neue Ideen, ja für Utopien in der Medizin, sie haben in ihrer Vergangenheit mit den Naturwissenschaften und der Mathematik eher auf Kriegsfuss gestanden, konventionelle Karrieremuster sind weniger attraktiv und der Ausgang der eigenen Behandlung wiegt mehr als die Sicherheit kollektiver Erfahrungen wie etwa klinischer Versuche.

\section{Literatur}

1 Bühring M: Zum Begriff «Komplementärmedizin». Forsch Komplementärmed 1999;6:125-126.

2 Meyer-Abich KM: Naturheilkunde oder Komplementärmedizin? Forsch Komplementärmed 1999;6:244-246.

3 Linde K: Das Forschende in der Komplementärmedizin. Forsch Komplementärmed 1999;6:293.
4 Fulder S, Munro R: The Status of Complementary Medicine in the UK, London, Threshold Foundation, 1981.

5 Fulder S, Munro R: Complementary medicine in the UK: Patients, practitioners and consultations. Lancet 1985;ii:542-545.

6 Wharton R, Lewith G: Complementary medicine and the general practitioner. BMJ 1986;292:14981500.

7 Fleck L: Entstehung und Entwicklung einer wissenschaftlichen Tatsache. Frankfurt/M., Suhrkamp, 1993. 